\title{
Young Children Have Difficulty Predicting Future Preferences in the Presence of a Conflicting Physiological State
}

\author{
Caitlin E. V. Mahy* \\ Department of Psychology, Brock University, St. Catharines, ON, Canada
}

This study examined children's predictions about their future preferences when they were in two different physiological states (thirsty and not thirsty). Ninety 3- to 7-year-olds were asked to predict what they would prefer tomorrow: pretzels to eat or water to drink after having consumed pretzels, and again after having had the opportunity to quench their thirst with water. Results showed that although children initially preferred pretzels to water at baseline, they more often indicated that they would prefer water the next day after they had consumed pretzels. After consuming water, however, the same children indicated they would prefer pretzels the next day. Children's verbal justifications for their choices rarely made reference to their current or future states, but rather justifications were more likely to make reference to their general preferences when they were no longer thirsty compared to when they were thirsty. Results suggest that current physiological states have a powerful influence on future preferences. The findings are discussed in the context of the development of episodic foresight, the Bischof-Kohler hypothesis, and the important and often overlooked role that children's current states play in future decision making. Copyright (c) 2015 John Wiley \& Sons, Ltd.

Key words: episodic foresight; future preferences; future self; conflicting states; young children

Episodic Foresight $(\mathrm{EpF})$, the ability to project oneself into future episodes, is a critical skill that children use in their everyday life. The importance of this ability to children's daily functioning has been reflected in the increased research interest in this area over the past 10 years (e.g. Atance \& O'Neill, 2005; Hudson,

*Correspondence to: Caitlin E. V. Mahy, Department of Psychology, Brock University, 500 Glenridge Avenue, St. Catharines, ON L2S 3A1, Canada. E-mail: caitlin.mahy@brocku.ca 
Mayhew, \& Prabhakar, 2010; Suddendorf, Nielsen, \& von Gehlen, 2011). Whether it is asking children to predict their needs on future hikes or trips to the desert (e.g. Atance \& Meltzoff, 2005) or to choose an item that will have utility in the future (e.g. Suddendorf et al., 2011), there are several established EpF tasks appropriate for young children and developmental trends in performance have been described.

Notably, there are gains in EpF performance during early childhood with most studies documenting increases in performance between 3 and 5 (although see Metcalf \& Atance, 2011; Payne, Taylor, Hayne, \& Scarf, 2015). Less work has examined EpF beyond age 5, but recent attempts have been made to extend our knowledge of EpF performance into middle childhood (Mahy, Grass, Wagner, \& Kliegel, 2014; Mahy, Atance, Moses, \& Kopp, under review). Although most EpF tasks show the most substantial gains in the preschool years, there are exceptions with children as old as 7 performing no better than 3-year-olds on certain tasks where current states directly conflict with future states (Mahy et al., 2014).

In daily life, children regularly anticipate future states in the face of a current, often conflicting state. For example, a child may have to anticipate the need for an umbrella given predicted rain later in the day despite currently sunny weather. Similarly, a child might have to anticipate later hunger in order to understand why she needs to take her lunch bag to school even though she is not currently hungry after eating a large breakfast. There is an important distinction to be made between these two examples, however. While the first example involves thinking about a future state that does not align with the current state, the second example is a particularly powerful because it involves conflict between a current and future physiological state. More specifically, the BischofKohler hypothesis (Bischof-Kohler, 1985) posits that non-human animals cannot act based on imagined future events but only act based on current needs (particularly physiological needs). Although this hypothesis has been challenged by findings that non-human animals can anticipate future events that involve satisfying a not currently experienced need (e.g. Correia, Dickinson, \& Clayton, 2007; Naqshbandi \& Roberts, 2006; Raby, Alexis, Dickinson, \& Clayton, 2007), it has been assumed that both adults and children have a superior ability to act based on future needs or desires. However, research on children's EpF suggests that young children have difficulty anticipating future physiological states in the face of a different current physiological state (Atance \& Meltzoff, 2006; Mahy et al., 2014), which begs the question of whether this difficulty applies to young children as it is posited to apply to non-human animals.

An important aspect of children's EpF that has been somewhat overlooked is that current states regularly conflict with future states in daily life (although see Suddendorf \& Corballis, 2007). Bélanger, Atance, Varghese, Nguyen, and Vendetti (2014) recently addressed this issue by asking children to predict future preferences that conflicted with current preferences. Children aged 3 to 5 were asked to predict their adult preferences that were often in conflict with their current preferences (whether they would prefer Kool-Aid or coffee when they were all grownup). Young children had difficulty predicting that their own future preference would be the typical adult preference (coffee), but had an easier time predicting that another child would have this adult preference when they were grown up. This study was a first step in examining the influence of current preferences that conflict with future preferences, but lacked a manipulation of children's states in order to have a current preference in direct conflict with a future preference. A manipulation design could further establish causality of a current state on future thinking. 
While much of the literature on children's EpF requires children to anticipate their future needs that may differ from their current needs (e.g. Atance \& Meltzoff, 2005; Suddendorf et al., 2011), an EpF task exists that directly pits children's future state against their current physiological state. Atance and Meltzoff (2006) first introduced the 'Pretzel task' where children were asked to make a baseline choice between pretzels and water. Not surprisingly, the majority of children between 3 and 5 preferred pretzels to water. Then, children were given the opportunity to eat a serving of pretzels while the experimenter read them a story (to induce thirst). After the story was finished and the pretzels were taken away, children were asked to make a prediction for their future self: what would they want if they returned to the lab tomorrow, pretzels to eat or water to drink? After consuming pretzels, most children responded that they would prefer water to pretzels in the future (in contrast with their baseline preference for pretzels). After answering this question, children were offered water to drink and the amount of water that was drunk was measured as an index of the child's level of thirst. The amount of water children drank was greater in the pretzel condition compared to a control condition where children ate no pretzels, suggesting that level of thirst was influencing children's future decision making. Importantly, children in the control condition continued to predict that their future selves would prefer pretzels to water. These findings suggest that preschoolers' future predictions for their preferences are powerfully affected by their current state and suggest that the Bischof-Kohler hypothesis may apply to young children when it comes to powerful physiological drives such as thirst.

However, an alternate explanation exists for Atance and Meltzoff's (2006) findings. It is possible that after consuming pretzels, children simply shifted their future preference to water because of 'taste boredom' for pretzels rather than their current state of thirst. Given that children in the control condition did not consume pretzels and therefore would not have experienced taste boredom, one would expect these children to continue to report a preference for pretzels. This alternate explanation could be ruled out by asking children to again predict their future preference after they are offered water. If taste boredom is driving responses, then children should continue to report preferring water to pretzels even after drinking water, as water would not completely override taste boredom for pretzels as taste boredom tends to persist over a longer period of time (e.g. Essed et al., 2006). In contrast, if their current state of thirst is what is leading them to predict that they will prefer water in the future, providing children with water (and thus quenching thirst) should result in children's responses returning to their baseline preference for pretzels over water. These predictions will be tested in the current study.

Although many EpF tasks require children to justify their future choices (e.g. Atance \& Meltzoff, 2005), no research has examined children's justifications for future preferences in the Pretzel task. Children's reasoning behind their preferences should provide additional insight into children's thinking about their future preferences especially when their preferences have changed over time based on an experimental manipulation. Children's justification of their choices (e.g. why would you want pretzels/water tomorrow?) should show whether children are making future choices based on their predicted future state, their current state, their general preferences, or some other factor. Although it is assumed that children's current state of thirst is leading them to choose water for their future selves, these explanations would be interesting especially given the fact that young children often have a shaky understanding of desires that arise from physiological states (Moses, Coon, \& Wusinich, 2000) and have difficulty understanding conflicting desires (Wright Cassidy et al., 2005). Therefore, children's explanations for their 
future choices in the Pretzel task may add a valuable source of information about how their current states might affect their future preferences. Making reference to their future states or general preferences would seem to indicate superior EpF compared to references to their current states or to other irrelevant factors. Thus, in a state of thirst one might expect children to provide explanations that reference their current state or irrelevant information (less future oriented responses), however, after the thirst is quenched one would expect children's explanations to reference general preferences or future states (more appropriate responses).

Given that past work has demonstrated the difficulty young children have with maintaining a consistent preference in the presence of a conflicting physiological state (i.e. maintaining a preference for pretzels over water when they are thirsty), the current study aimed to examine the effect of two manipulations of physiological state on children's EpF. The first, a replication of Atance \& Meltzoff, required children to choose between pretzels and water for the future after consuming pretzels, and the second required children to again choose between pretzels and water for their future self after they had been given a chance to quench their thirst with water. The goal of these two manipulations was to address the possibility that children may have shifted their future preference to water over pretzels because of taste boredom for pretzels rather than because of the influence of their current state of thirst on their future preferences. Novel to this study, children were asked to provide explanations for their choices in order to gain further insight into their $\mathrm{EpF}$ and, more specifically, whether they referred to their future states, current states, general preferences, or irrelevant information in their justifications.

The predictions of the current study were as follows: (1) despite having stated an initial preference for pretzels, 3- to 7-year-old children will be more likely to chose water as their future preference when they are thirsty, (2) after children have been given the opportunity to drink water their future preference will return to pretzels (in line with their baseline preference), and (3) children's explanations of their future preferences will be less likely to refer to their general preferences and less likely to contain relevant information (and thus fall into the 'other' category) when they are thirsty, but more likely to refer to their general preferences after they have had the chance to drink water and their current state is consistent with their baseline state.

\section{METHOD}

\section{Participants}

Participants were 100 typically developing children aged three to seven (25 3year-olds; 22 4-year-olds; 18 5-year-olds; 19 6-year-olds; and 19 7-year-olds). Four children were uncooperative and did not produce useable data. An additional six children ate very few pretzels during the Pretzel Task (less than 8) so their data were excluded, as one could not be sure that thirst had been induced by the manipulation. The final sample consisted of 90 children: 18 3-year-olds (9 girls; $M=42.89$ months, $S D=3.18$ ), 18 4-year-olds, (9 girls; $M=52.78$ months, $S D=3.77$ ), 18 5-year-olds (9 girls; $M=67.00$ months, $S D=3.01$ ), 18 6-year-olds (9 girls; $M=78.56$ months, $S D=3.20$ ), and 187 -year-olds (9 girls; $M=88.72$ months, $S D=3.69)$. Children were mostly Caucasian and from a middle class background consistent with the general populations from which they were drawn. Participants were recruited from university participant databases at the University of Oregon and the University of Ottawa. 


\section{Measure}

Pretzel Task (adapted from Atance \& Meltzoff, 2006). Children were offered a small amount of apple juice (approximately $3 \mathrm{oz}$.) at the beginning of this task to ensure they were not thirsty at baseline. After children had the opportunity to drink some juice, it was removed. The experimenter asked what the child preferred to have right now: pretzels to eat or water to drink. Children were simultaneously shown a photograph of a bowl of pretzels and a bottle of water, and their responses were recorded. Regardless of children's initial stated preference, the experimenter told them that it was time to listen to a story and that they could eat as many pretzels as they liked during this time. A small bowl with 36 pretzel sticks was placed in front of the child while the experimenter read a story ('The Perfect Picnic'; Maestro, 1986), which took approximately $6 \mathrm{~min}$.

After the story was finished, the experimenter removed the bowl of pretzel sticks. Then, the experimenter asked children to pretend that they were going to come back to the laboratory the next day to play a marble game (children were shown a photograph of the marble game). It was emphasized that children were not going to play with the marble game today, but needed to imagine playing with it tomorrow. Children were shown the photograph of a bowl of pretzels and a bottle of water and were asked what they would like to have during the marble game tomorrow: pretzels to eat or water to drink? After children had responded, they were asked: 'why would you like pretzels/water tomorrow?' After these test questions were asked, children were offered $9 \mathrm{oz}$. of water to drink. The amount of water (0-9 oz.) children drank was measured and recorded (as a measure of how thirsty they were), as was the number of pretzels children ate (to ensure that they were thirsty when they were answering the questions about the future). After children had been given the opportunity to drink water, they were asked again to imagine coming back to the lab the next day and were asked what would they like to have tomorrow while playing the marble game: pretzels or water?

\section{Offline Coding Procedures}

Children's responses to justify their choices were first scored offline as referring to a future state (score of 1 ) or not referring to a future state (score of 0 ) and second categorized into whether they referred to a future state, current state, general preference, or another category. Inter-rater agreement between two raters was substantial for whether the response was future-oriented or not (Cohen's kappas > .68) and for the categorization of responses (Cohen's kappas $>.80$ ) for justifications after they had consumed pretzels and after they had consumed water.

Two separate analyses were conducted: one including responses from all children $(\mathrm{N}=90)$, and another including only responses from children who initially preferred pretzels to water $(\mathrm{N}=69)$. Children were given a score of 1 if they indicated that they would prefer pretzels tomorrow and a score of 0 if they said that they would prefer water (both for responses after consuming pretzels and after consuming water).

\section{Procedure}

Children participated in the Pretzel task as a part of a larger study on the development of episodic foresight (Mahy et al., under review). Given the detail necessary to analyse children's three choices between pretzels and water across three time points and their explanations for their choices, this task was analysed separately in 
the current manuscript. The Pretzel task was the first task administered and took approximately $10 \mathrm{~min}$. Parents were asked to fill out a demographics questionnaire while children participated in the study. At the end of the session, children were thanked and were given a small prize for their participation. The research ethics boards at University Oregon and University Ottawa approved all procedures.

\section{RESULTS}

Table 1 shows the number of children that chose pretzels or water for their present self at baseline, for their future selves after they consumed pretzels, and for their future selves after they consumed water.

At the start of the study, $78 \%$ of children drank the juice that was offered to them $(M=.778, S D=.418)$. Children on average ate a large number of pretzel sticks $(M=16.7, S D=8.85)$ and drank a small amount of water in oz. when it was offered to them after they had eaten pretzels $(M=1.89, S D=1.95)$.

Because of the categorical outcome (pretzels or water), a repeated measures logistic regression with age group (3- vs. 4- vs. 5- vs. 6- vs. 7-year-olds) as a between subjects predictor and state manipulation (baseline vs. future self after pretzels vs. future self after water) as a within-subjects predictor was carried out on all children's predicted preference of pretzels or water. Age group was not a significant predictor of children's performance $(p=.662)$. State manipulation, however, was a significant predictor of children's performance, Wald $\chi^{2}(2)=41.84, p<.001$. Children were more likely to judge that they would prefer water over pretzels in the future after they had consumed pretzels $(67.8 \%$ preferred water) compared to their baseline preference $(23.3 \%$ preferred water; Odds ratio $=6.91)$ but were no more likely to judge that they would prefer water over pretzels in the future after they had consumed water (22.1\% preferred water) compared to their baseline preference $(23.3 \%$ preferred water; Odds ratio $=.93$; see Figure $1 \mathrm{~A})$.

When only the children who preferred pretzels at baseline were included in the analyses $(\mathrm{N}=69)$, a similar pattern of results emerged (see Figure 1B). A repeated measures logistic regression with age group (3- vs. 4- vs. 5- vs. 6- vs. 7-year-olds) as a between subjects predictor and state manipulation (future self after pretzels vs. future self after water) as a within-subjects predictor was carried out on children's predicted preference of pretzels or water. Age group was not a significant predictor of children's performance $(p=.952)$. State manipulation emerged as a significant predictor of children's future judgments, Wald $\chi^{2}(1)=35.04, p<.001$. Children were more likely to prefer pretzels to water for their future self after consuming water (85.1\% preferred pretzels) than after consuming pretzels $(31.9 \%$ preferred pretzels; Odds ratio $=7.42)$.

The amount of water children drank was positively correlated with the number of pretzels they consumed, $r(69)=.32, p=.007$. Children who indicated that they would choose water for their future selves (after consuming pretzels) also drank more water when they were offered some, $r(69)=-.293, p=.015$, suggesting that these children who chose water over pretzels after consuming pretzels were thirstier than those who chose pretzels over water. In contrast, the amount of water drank was not related to children's choice for their future self after consuming water, $r(67)=.03, p=.83$. The number of pretzels that children ate was unrelated to their decisions about their future preferences, $r s(67)<.03, p s>.586$.

Children's responses to justify why they selected pretzels or water for their future selves were elicited both after consuming pretzels and after drinking water. Initially, these responses were coded as future oriented or not. Results suggested 


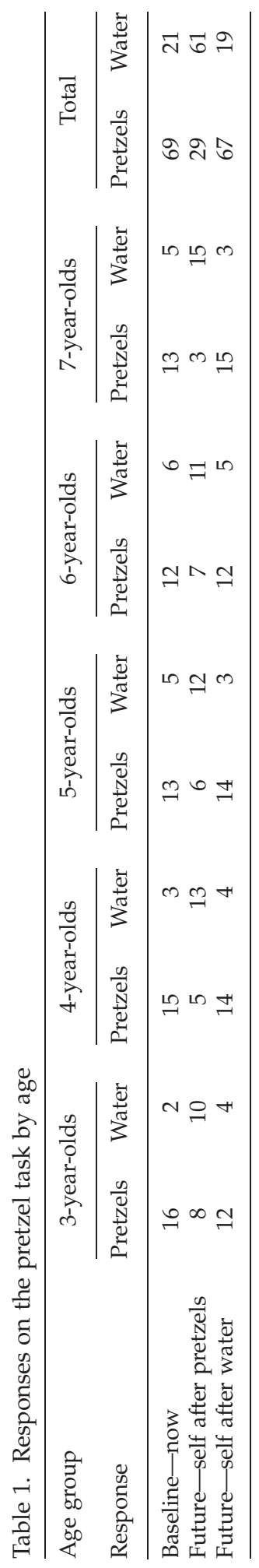




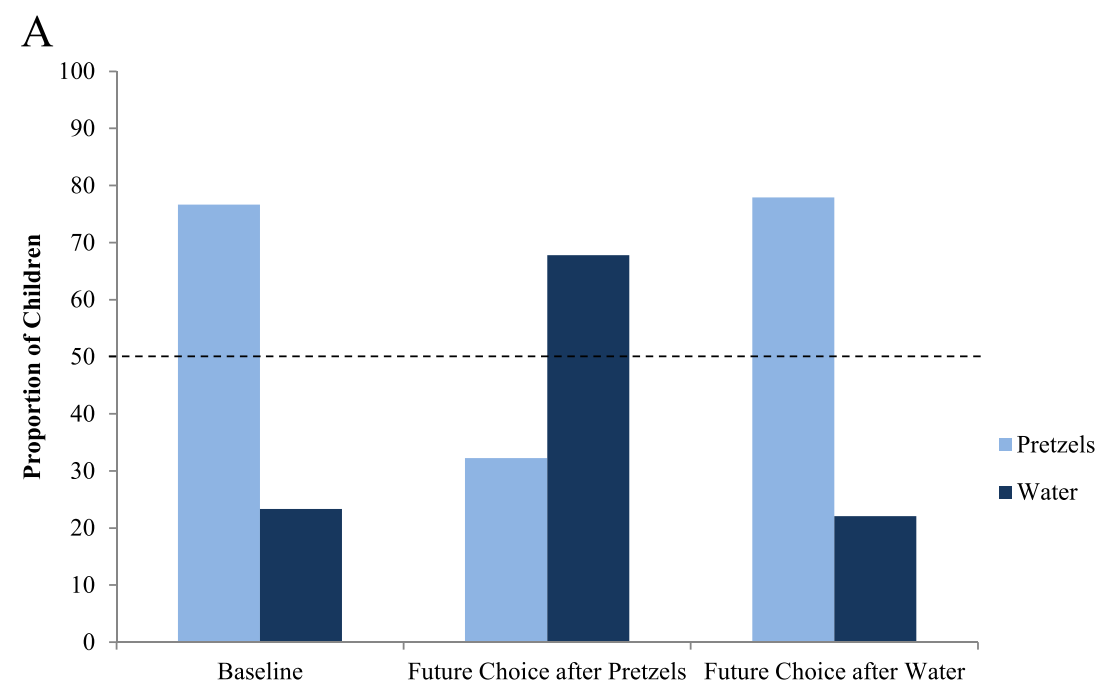

B

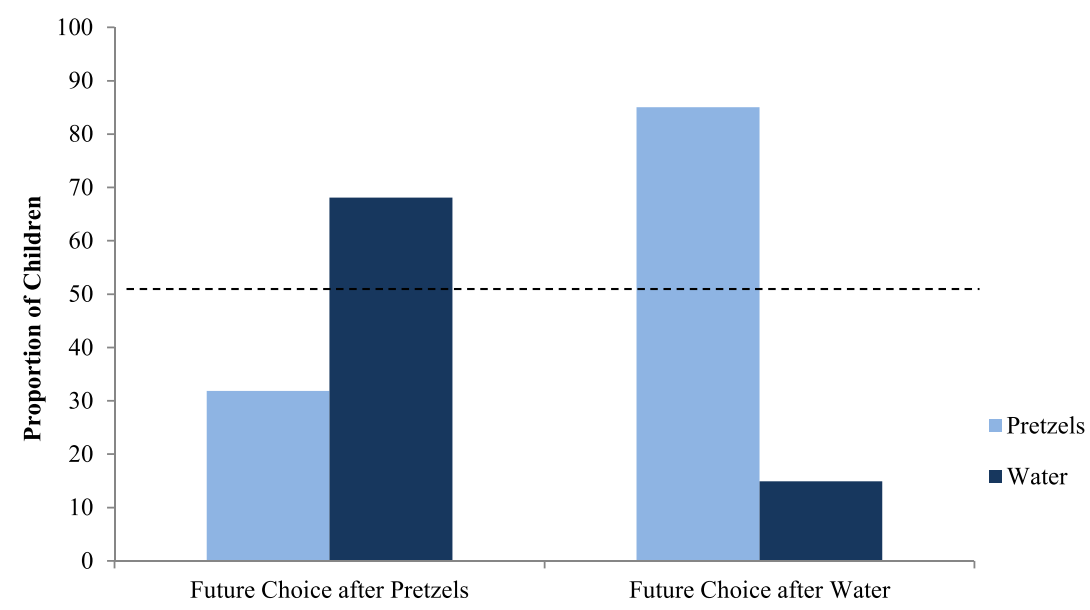

Figure 1. Percentage of children preferring pretzels at baseline, for their future self after eating pretzels, and for their future self after consuming water for full sample (A) and only for children who preferred pretzels at baseline (B). Dashed line represents chance responding.

that a minority of children referred to the future in their responses after consuming pretzels $(21.0 \%)$ and after consuming water $(16.9 \%), \chi^{2} s(1)>20.90, p s<.001$. When entered into a binary regression, surprisingly, children's age in months was not a significant predictor of whether they made a future-oriented response or not, Wald $\chi^{2}(1)<.08$, $p$ s $>.770$.

To gain additional insight into the nature of children's justifications, responses were then coded into one of four categories based on whether responses made reference to: (1) a future state, (2) a current state, (3) a general preference, or (4) something not covered by the first three categories (other). Table 2 provides the number of children whose explanations were coded into the four different categories and Table 3 provides sample answers that fell into each category. Children's responses were equally distributed among the four categories after consuming 
Table 2. Children's justifications for their future preferences by category

\begin{tabular}{lcc}
\hline & After consuming pretzels & After consuming water \\
\hline Future state & 13 & 11 \\
Present state & 15 & 17 \\
General preference & 20 & 32 \\
Other & 14 & 5 \\
\hline
\end{tabular}

Note—results are only shown for children who preferred pretzels at baseline.

pretzels, $\chi^{2}(3)=1.87, p=.60$. In contrast, after consuming water, children's responses were no longer equally distributed, $\chi^{2}(3)=24.79, p<.001$. Follow-up analyses using a single variable goodness of fit test showed that, after consuming water, children's responses were more likely to fall into the category of 'general preference', $\chi^{2}(1)=15.26, p<.001$, and less likely to fall into the 'other' category, $\chi^{2}(1)=7.79, p=.005$, than would be expected based on a even distribution across the four categories.

\section{DISCUSSION}

The current study provides further evidence that manipulating current states can influence children's judgments about future preferences and rules out the possibility that previous results (Atance \& Meltzoff, 2006) were because of children's taste boredom for pretzels. The results show that children, even after stating a baseline preference for pretzels over water, indicate their future preference is water when they are thirsty but will revert back to their general preference for pretzels after consuming a small amount of water. This study demonstrates that children's future thinking is affected by their current states and these states can be manipulated with direct effects on children's future choices. Although there is debate on whether the Bischof-Kohler hypothesis holds for non-human animals, our results suggest that it may apply to young children as they seem to struggle to anticipate future states in the face of a current one that is in conflict with that future state. More broadly, this suggests that young children, like adults, have a 'presentism' or 'projection' bias (e.g. Gilbert, Gill, \& Wilson, 2002; Loewenstein \& Angner, 2003) and supports predictions from the Bischof-Kohler hypothesis that current physiological states powerfully influence future preferences. This is the first study to examine children's justifications for their future choices in the Pretzel task. Findings revealed that in their verbal explanations for future choices children rarely make reference to the future (or current states for that matter). Further, after consuming pretzels, children's justifications for their future choices were distributed evenly across the four categories. In contrast, after consuming water, children were more likely to explain their future choice with reference to general preferences (a more optimal justification) and less likely to explain their choice for their future self using justifications that fit into the 'other' category indicating an unusual or nonsensical response.

Taken together with findings by Atance and Meltzoff (2006) that showed that children who were not given pretzels continued to state that their future preference would be pretzels, the current study suggests that young children's future thinking is strongly influenced by their current state. Further, these results rule out the taste boredom alternative account that could have led to Atance and Meltzoff's (2006) findings as children's preference returned to pretzels after they 


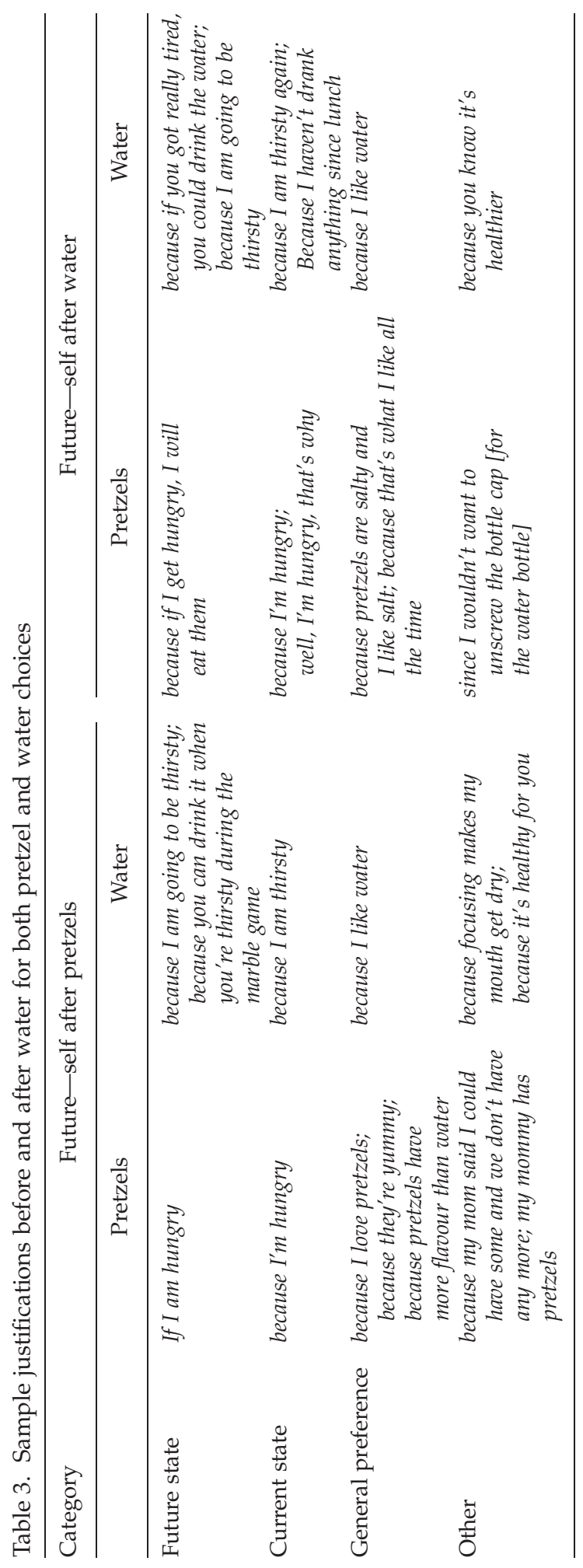


consumed a small amount of water, suggesting that the state of thirst was driving them to select water as a future preference rather than being tired of pretzels. Present results suggest that current states can be manipulated in the laboratory and have distinct influences on children's future thinking. A thirsty child is more likely to predict that they will prefer water the next day and a child whose thirst has just been quenched is more likely to predict that they will prefer pretzels the next day, even though just minutes earlier, in a state of thirst, many children had indicated they would prefer water the next day. The states manipulated in this study were physiological in nature (thirst/satiation) but it would be interesting for future studies to examine the impact of conflicting emotional or knowledge states on children's EpF. It is possible that physiological states such as thirst and hunger have a special status that might have an especially powerful impact on thinking about future preferences compared to non-physiological states (see Bischof-Kohler, 1985).

In contrast to most EpF tasks that do not involve physiological states (Atance \& Meltzoff, 2005; Suddendorf et al., 2011), there were no age-related increases in Pretzel task performance, suggesting that even 7-year-olds performed as poorly as 3-year-olds. This is consistent with previous work that failed to find age differences in Pretzel task performance between 3 and 7 (Mahy et al., 2014). It is possible that given the salience of thirst as a physiological state, older children and even adults struggle to reason about the future accurately when they are under the influence of such a powerful motivational state. This begs the question of when in development are children able to accurately reason about future preferences in the face of a conflicting current state? Research with adults suggests that predictions about a hiker's thirst or hunger was influenced by the participant's own exercise-induced thirst (Van Boven \& Loewenstein, 2003), suggesting that a salient current state has a strong influence on social projections. Therefore, future projections that are in conflict with a current state may be something that even adults struggle with (Wilson \& Gilbert, 2003) as in the classic study that showed that hungry individuals buy more food at the supermarket compared to individuals who had recently eaten (Nisbett \& Kanouse, 1969).

Novel to this study, children were asked to explain their future preferences. Less than half of children made reference to their future or current state. Curiously, children who indicated that they would prefer water the next day after eating pretzels (and thus, were influenced by their current state of thirst given their baseline preference for pretzels) did not tend to make reference to their current state of thirst suggesting that children lack the awareness that their preferences are being influenced by their current state. Although one might expect thirsty children to make reference to their current thirst when making a choice about the future, if the current state is influencing their future thinking, these findings are consistent with a very general difficulty that young children have with understanding one's own states and motivations (Flavell, 1999, 2000; Gopnik \& Slaughter, 1991). Thus, children might not make reference to their current state because they are unaware that it is influencing them (as their explanations suggest) or that they are aware that their current state should not influence future preference (which seems unlikely given that their choice for water is influenced by their current state). It seems that although their current state clearly influences their future preferences, children's explanations indicate that they lack the awareness that their future choices are being influenced by their current states or even future states. Further, when children were thirsty (after consuming pretzels) their explanations were not more likely to fall into any one of the four categories than another. In contrast, very few children's explanations fell into the 'other' category and many more fell into the 
general preference category when they were justifying their future prediction after consuming water. This makes sense given that children's future preference after consuming water (pretzels) was in line with their baseline general preference. Interestingly, the decrease in other responses after consuming water likely reflects the fact that children could rely on their general preferences to justify the response "pretzels," whereas in a state of thirst children often used non-preference related justifications (e.g. because focusing makes my mouth get dry) or health-based justifications (e.g. because water is healthy for you) for selecting water that fit into the 'other' category although some children still rely on general preferences.

An interesting addition to the current study would have been to ask children to justify their baseline preferences. One would suspect that children's baseline preferences would have been explained with reference to their general preferences or perhaps a current state such as hunger, but this would have been useful in assessing changes in explanation across time. Future research in EpF should not overlook the importance of asking children to explain their choices as it offers further insight into their future thinking or their lack of future thinking (at least in terms of their justifications being future oriented).

A potential alternate explanation for these findings is that after drinking water children may have experienced water taste boredom and thus switched their future preference back to pretzels after having had the chance to drink water. This explanation is unlikely for four reasons. First, children drank a very small amount of water equivalent to a few sips unlikely to make them bored of water (but enough to quench thirst). Second, the amount of water that children drank was unrelated to their predicted choice for pretzels or water for their future self. According to the water boredom account, children who drank more water should have been more likely to choose pretzels if their choice was driven by the amount of water they consumed, which was clearly not the case. Third, no children made reference to being tired of water in their justifications for their choices for pretzels after consuming water. Finally, past research has suggested that children have a bias for consistency (rather than inconsistency) between current and future preferences even when they are unlikely (Bélanger et al., 2014). An important future step will be to further rule out the possibility that children shift their future preferences toward the opposite choice of their current preference, however, given that Atance and Meltzoff's (2006) findings showed that children in the control condition who did not have the opportunity to eat pretzels continued to prefer pretzels for the future self, it seems unlikely that children shift their preferences in the absence of a current physiological state.

Given that physiological states seem to have a particularly powerful influence on future thinking, future work could examine whether these motivational states could be reduced or even reversed using top-down reasoning. For example, one could have children make statements about future states while experiencing a conflicting state in the present.

In sum, children's future preferences are influenced by their current states and these preferences can change quickly in response to a change in a current physiological state. In fact, when it comes to physiological states, children seem to not engage in $\mathrm{EpF}$ and rather respond based on their current states. Interestingly, our justification data suggest that children are unaware of this, as they do not tend to make more references to their current state when they were thirsty. These findings have important implications for children's EpF and how it is measured. For optimal EpF accuracy, it is critical that young children are not under the influence of a physiological state such as thirst that may be in conflict with their future states. In contrast, more accurate EpF occurs when current states align with future 
states. Future work should address the question of whether current physiological states can be overcome to engage in $\mathrm{EpF}$ about future states, particularly in young children. EpF is a critical ability to master during the childhood years and the ability to overcome conflict between current and future states may be an important developmental achievement that results in increased (but likely imperfect) accuracy in future thinking and planning more generally.

\section{ACKNOWLEDGEMENTS}

I wish to thank Michele Anderson, Prachi Bhuptani, Leia Kopp, Jack Rossing, and Madeline Weissman for assistance with data collection and Tristan Fassel for assistance with data coding. I am grateful to Cristina Atance and Laura Batterink for comments on previous versions of this manuscript.

\section{REFERENCES}

Atance, C. M., \& Meltzoff, A. N. (2005). My future self: Young children's ability to anticipate and explain future states. Cognitive Development, 20, 341-361. doi: 10.1016/j. cogdev.2005.05.001

Atance, C. M., \& Meltzoff, A. N. (2006). Preschoolers' current desires warp their choices for the future. Psychological Science, 17, 583-587. doi: 10.1111/j.1467-9280.2006.01748.x

Atance, C. M., \& O'Neill, D. K. (2005). The emergence of episodic future thinking in humans. Learning and Motivation, 36, 126-144.

Bélanger, M. J., Atance, C. M., Varghese, A. L., Nguyen, V., \& Vendetti, C. (2014). What will I like best when I'm all grown up? Preschoolers' understanding of future preferences. Child Development, 85, 2419-2431.

Bischof-Kohler, D. (1985). Zur phyogenese menschlicher motivation [On the phylogeny of human motivation]. In L. H. Eckensberger, \& E. D. Lantermann (Eds.), Emotion und reflexivitut [Emotion and reflexivity] (pp. 3-47). Vienna: Urban \& Schwarzenberg.

Correia, S. P., Dickinson, A., \& Clayton, N. S. (2007). Western scrub-jays anticipate future needs independently of their current motivational state. Current Biology, 17, 856-861.

Essed, N. H., van Staveren, W. A., Kok, F. J., Ormel, W., Zeinstra, G., \& de Graaf, C. (2006). The effect of repeated exposure to fruit drinks on intake, pleasantness and boredom in young and elderly adults. Physiology \& Behavior, 89, 335-341.

Flavell, J. H. (1999). Cognitive development: Children's knowledge about the mind. Annual Review of Psychology, 50, 21-45.

Flavell, J. H. (2000). Development of children's knowledge about the mental world. International Journal of Behavioral Development, 24, 15-23.

Gilbert, D. T., Gill, M. J., \& Wilson, T. D. (2002). The future is now: Temporal correction in affective forecasting. Organizational Behavior and Human Decision Processes, 88, 430-444.

Gopnik, A., \& Slaughter, V. (1991). Young children's understanding of changes in their mental states. Child Development, 62, 98-110.

Hudson, J. A., Mayhew, E. M., \& Prabhakar, J. (2010). The development of episodic foresight: Emerging concepts and methods. Advances in Child Development and Behavior, 40, 95-137.

Loewenstein, G., \& Angner, E. (2003). Predicting and indulging changing preferences. In G. Loewenstein, D. Read, \& R. Baumeister (Eds.), Time and decision: Economic and psychological perspectives on intertemporal choice (pp. 351-391). New York: Russell Sage Foundation.

Maestro, B. (1986). The perfect picnic. Racine, WI: Western Publishing Company, Inc.

Mahy, C. E. V., Atance, C. M., Moses, L. J., \& Kopp, L. (under review). Developmental trajectories of episodic foresight for self and other in early childhood. 
Mahy, C. E. V., Grass, J., Wagner, S., \& Kliegel, M. (2014). These pretzels are going to make me thirsty tomorrow: Differential development of hot and cool episodic foresight in early childhood? British Journal of Developmental Psychology, 32, 65-77.

Metcalf, J. L., \& Atance, C. M. (2011). Do preschoolers save to benefit their future selves?. Cognitive Development, 26, 371-382.

Moses, L. J., Coon, J. A., \& Wusinich, N. (2000). Young children's understanding of desire formation. Developmental Psychology, 36, 77-90.

Naqshbandi, M., \& Roberts, W. A. (2006). Anticipation of future events in squirrel monkeys (Saimiri sciureus) and rats (Rattus norvegicus): Tests of the Bischof-Kohler hypothesis. Journal of Comparative Psychology, 120, 345-357.

Nisbett, R. E., \& Kanouse, D. E. (1969). Obesity, food deprivation, and supermarket shopping behavior. Journal of Personality and Social Psychology, 12, 289-294.

Payne, G., Taylor, R., Hayne, H., \& Scarf, D. (2015). Mental time travel for self and other in three-and four-year-old children. Memory, 23, 675-682. doi: 10.1080/09658211.2014. 921310

Raby, C. R., Alexis, D. M., Dickinson, A., \& Clayton, N. S. (2007). Planning for the future by western scrub-jays. Nature, 445, 919-921.

Suddendorf, T., \& Corballis, M. C. (2007). Mental time travel across the disciplines: The future looks bright. Behavioral and Brain Sciences, 30, 335-345.

Suddendorf, T., Nielsen, M., \& von Gehlen, R. (2011). Children's capacity to remember a novel problem and to secure its future solution. Developmental Science, 14, 26-33. doi: 10.1111/j.1467-7687.2010.00950.x

Van Boven, L., \& Loewenstein, G. (2003). Social projection of transient drive states. Personality and Social Psychology Bulletin, 29, 1159-1168.

Wilson, T. D., \& Gilbert, D. T. (2003). Affective forecasting. Advances in Experimental Social Psychology, 35, 345-411. doi:10.1016/S0065-2601(03)01006-2

Wright Cassidy, K., Cosetti, M., Jones, R., Kelton, E., Meier Rafal, V., Richman, L., \& Stanhaus, H. (2005). Preschool children's understanding of conflicting desires. Journal of Cognition and Development, 6, 427-454. 